\title{
Morphing of Amphipathic Helices to Explore the Activity and Selectivity of Membranolytic Antimicrobial Peptides
}

\section{Journal Article}

Author(s):

Müller, Alex T.; Posselt, Gernot; Gabernet, Gisela; Neuhaus, Claudia; Bachler, Simon; Blatter, Markus; Pfeiffer, Bernhard; Hiss, Jan A.; Dittrich, Petra S. (D); Altmann, Karl-Heinz; Wessler, Silja; Schneider, Gisbert

\section{Publication date:}

2020-10-06

Permanent link:

https://doi.org/10.3929/ethz-b-000449945

Rights / license:

Creative Commons Attribution-NonCommercial-NoDerivatives 4.0 International

Originally published in:

Biochemistry 59(39), https://doi.org/10.1021/acs.biochem.0c00565

\section{Funding acknowledgement:}

157190 - Experiment-guided computational de novo exploration of peptide-membrane interaction (SNF) 160699 - Engineering the Targeted Drugs of the Future: A General Approach (SNF) 


\section{Morphing of Amphipathic Helices to Explore the Activity and Selectivity of Membranolytic Antimicrobial Peptides}

Alex T. Müller, Gernot Posselt, Gisela Gabernet, Claudia Neuhaus, Simon Bachler, Markus Blatter, Bernhard Pfeiffer, Jan A. Hiss, Petra S. Dittrich, Karl-Heinz Altmann, Silja Wessler, and Gisbert Schneider*

Cite This: Biochemistry 2020, 59, 3772-3781

Read Online

ACCESS | 네 Metrics \& More | 回 Article Recommendations | st Supporting Information

ABSTRACT: Naturally occurring membranolytic antimicrobial peptides (AMPs) are rarely cell-type selective and highly potent at the same time. Template-based peptide design can be used to generate AMPs with improved properties de novo. Following this approach, 18 linear peptides were obtained by computationally morphing the natural AMP Aurein 2.2d2 GLFDIVKKVVGALG into the synthetic model AMP KLLKLLKKLLKLLK. Eleven of the 18 chimeric designs inhibited the growth of Staphylococcus aureus, and six peptides were tested and found to be active against one resistant pathogenic strain or more. One of the peptides was broadly active against bacterial and fungal pathogens without exhibiting toxicity to certain human cell lines. Solution nuclear magnetic resonance and molecular dynamics simulation suggested an obliqueoriented membrane insertion mechanism of this helical de novo peptide. Temperature-resolved circular dichroism spectroscopy pointed to conformational flexibility as an essential feature of cell-type selective AMPs.
$\mathrm{T}$ he global increase in the prevalence of resistant bacteria is a threat to modern society, and the discovery of novel antibiotics that have not yet become susceptible to resistance is an urgent need. ${ }^{1}$ Membranolytic antimicrobial peptides (AMPs) could be a partial alternative to the current targetspecific antibiotic agents. ${ }^{2}$ A large variety of AMPs from natural sources have shown promising results as potential antibiotics with a direct mode of action on prokaryotic cell membranes as well as on mammalian cancer cells. ${ }^{3,4}$ Their direct interaction with membrane structures without the need for target proteins or receptors makes them less susceptible to a rapid onset of bacterial resistance and renders them potentially active against resistant strains. ${ }^{5}$ More than 3000 naturally occurring AMPs have been described and compiled in databases. ${ }^{6-8}$ These sequence collections offer a basis for template-based design and statistical analysis to develop novel AMPs. ${ }^{9}$ Several studies have revealed steep sequence-activity relationships between highly active and completely inactive peptides, with the possibility of a single-amino acid substitution abrogating antimicrobial activity (Table S1)..$^{10-12}$

Natural AMPs adopt a broad range of secondary structures and folds. ${ }^{2}$ We hereafter focus on short cationic $\alpha$-helical AMPs from the Anura order, namely, the Aurein and Citropin families. ${ }^{13,14}$ Amino acid substitutions in an Aurein AMP confirmed that subtle changes in the peptide sequence can have a profound impact on the peptide's activity. ${ }^{15}$ These antimicrobial activity changes correlated with a difference in structural flexibility between the studied peptides, specifically in their terminal portions. Similarly, a conformationally restricted analogue of an influenza virus fusion protein was less potent in promoting lipid mixing, a surrogate for the ability to lyse bacterial membranes. The flexibility of the fusion peptide was essential for the peptide's ability to destabilize the host membrane and promote transfection. ${ }^{16}$ In a related study, a diarylethene moiety was incorporated into the backbone of membrane-interacting peptides. ${ }^{17}$ By reversible isomerization of the linker, rigid and flexible peptide conformations were induced, and differences in their interactions with lipid membranes could be studied by biophysical methods. These previous studies suggest that the conformational degrees of freedom ("flexibility") of the amino acid residues may critically affect the activity and mechanism of action.

To gain deeper insight into the relationship between the amino acid sequence and the antimicrobial activity of AMPs, we performed a detailed analysis of the influence of sequence variation on the antimicrobial activity of an amphipathic $\alpha$ helical semisynthetic AMP from the Aurein peptide family. ${ }^{15}$ On the basis of published solution nuclear magnetic resonance (NMR) structures of Aureins, one can assume an inducible overall $\alpha$-helical secondary structure of many, if not all, members of the Aurein family. As not all Aureins are active AMPs, the question of whether a peptide's amino acid sequence pattern or slight changes in secondary structure are

Received: July 9, 2020

Revised: September 7, 2020

Published: September 16, 2020 
causative factors for antimicrobial activity arises. To investigate which of these features accounts for antimicrobial or cytotoxic activity, we performed sequence "morphing" with the aim of conserving an overall helical peptide structure while modifying the amino acid sequence, changing one residue at a time. As starting points, we selected two template peptides, both encompassing 14 residues: the C-terminally shortened Aurein $2.2[\mathrm{Au} 2.2 \mathrm{~d} 2$, sequence of GLFDIVKKVVGALG; Protein Data Bank (PDB) entry $5 \mathrm{mxl}]^{15,18}$ and the synthetic model AMP Klk14 (sequence of KLLKLLKKLLKLLK). ${ }^{19}$ These cationic peptides were selected as they both can form amphipathic helices with a central twin-lysine motif (Figure 1) and possess a common pharmacophore sequence pattern

a)

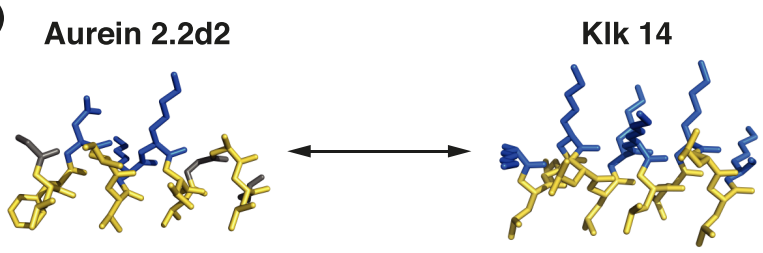

b)

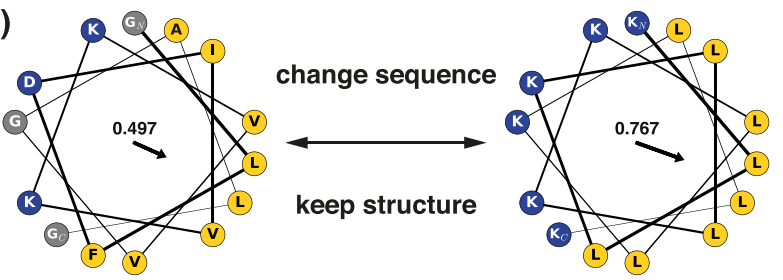

c)

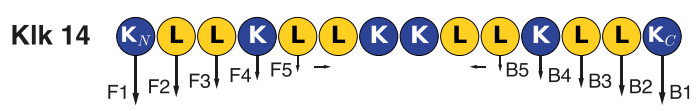

Aurein 2.2d2 G L F D I V K V V G A L G

Figure 1. Schematic representation of the morphing process. (a) Idealized helical structures [constructed with the "fab" command in PyMol (http://www.pymol.org)] for both templates. (b) Helical wheel plots of both template sequences showing amphipathicity quantified as hydrophobic moments [values and arrows, plotted with modlAMP (http://modlamp.org)] ${ }^{26}$ As both helices share the same orientation of their hydrophobic faces, sequential changes should not affect the overall amphipathicity. (c) Stepwise residue replacement between the two template sequences in forward ( $\mathrm{N}$ - to $\mathrm{C}$-terminus, denoted F) and backward (C- to N-terminus, denoted B) directions. Hydrophobic residues are colored yellow, and polar ones blue.

(PHHPHНPРHНPHHP, where $\mathrm{P}$ is a polar residue and $\mathrm{H}$ is a hydrophobic residue). By sequence morphing in both directions (from the $\mathrm{N}$ - to $\mathrm{C}$-terminus and from the $\mathrm{C}$ - to $\mathrm{N}$-terminus), we generated 18 chimeras of Au2.2d2 and Klk14 peptides. A schematic of the morphing approach is shown in Figure 1.

Template-based design approaches have already been pursued in AMP research. For example, Han et al. optimized the cationic helical AMP myxinidin by substituting selected residues with Arg, Lys, or Trp and thereby increased activity and selectivity. ${ }^{20}$ Similarly, the palindromic antimicrobial peptide Pa-MAP2 was designed on the basis of Pa-MAP by Franco and co-workers, revealing a well-organized $\alpha$-helix with flexible termini and profound interactions with phospholipids. ${ }^{21}$ Abdel Monaim et al. optimized the depsipeptide Teixobactin to obtain a cationic AMP. ${ }^{22}$ In contrast to these single-template design approaches, our method allows us to simultaneously morph one AMP into another AMP while retaining an overall helical structure of the intermediates. This concept enabled the systematic investigation of the sequence space between two known cationic helical AMPs and resulted in the discovery of relevant features for AMP activity and selectivity.

\section{METHODS}

Peptide Synthesis, Analytics, and Purification. Peptide sequences were synthesized by 9-fluorenylmethoxycarbonyl (Fmoc) solid-phase peptide synthesis ${ }^{23,24}$ on automated parallel peptide synthesizers (Symphony and Prelude, Gyros Protein Technologies) using $200 \mathrm{mM}$ Rink amide 4-methyl benzhydrylamine (MBHA) resin (AAPPTec) $\left(0.52 \mathrm{mmol} \mathrm{g}^{-1}\right)$ as a solid support, leading to C-terminally amidated peptides. All amino acids were purchased from AAPPTec and dissolved in dimethylformamide (DMF) (Sigma-Aldrich). Peptides were treated with $20 \%$ pyrrolidine (Acros Organics) in DMF (v/v) to deprotect the resins and the amino acids from Fmoc groups. Amino acids were subsequently coupled to the resin-bound peptides using $200 \mathrm{mM}$ O-(6-chlorobenzotriazol-1-yl)$N, N, N^{\prime}, N^{\prime}$-tetramethyluronium hexafluorophosphate (HCTU) (Protein Technologies) and $400 \mathrm{mM} \mathrm{N}$-methylmorpholine (NMM) (Fisher Chemical) as coupling reagents. After coupling of the terminal amino acids, peptides were washed with DMF and dichloromethane (DCM) (SigmaAldrich) and cleaved from the resin by a mixture of $95 \%$ trifluoroacetic acid (TFA) (ABCR), 2.5\% triisopropylsilane (TIS) (Sigma-Aldrich), and $2.5 \%$ doubly distilled $\mathrm{H}_{2} \mathrm{O}$ $\left({ }_{d d} \mathrm{H}_{2} \mathrm{O}\right)(\mathrm{v} / \mathrm{v} / \mathrm{v})$. Peptides were precipitated with diisopropyl ether (Fluka) at $-20^{\circ} \mathrm{C}$ overnight, centrifuged (for $10 \mathrm{~min}$ at $-10^{\circ} \mathrm{C}$ and $3000 \mathrm{rpm}$ ), and resuspended for four cycles in icecold diisopropyl ether and dried under an ambient atmosphere for 3 days. The crude peptides were purified using reversephase preparative high-performance liquid chromatography (HPLC) (Shimadzu) on a Nucleodur C18 HTec column (150 $\mathrm{mm} \times 21 \mathrm{~mm}, 5 \mu \mathrm{m}, 110 \AA$ ) with a linear 5 to $70 \%$ acetonitrile (Sigma-Aldrich) in water gradient containing $0.1 \%$ formic acid (Merck Millipore) and a flow rate of $0.5 \mathrm{~mL} \mathrm{~min}^{-1}$ (at $40{ }^{\circ} \mathrm{C}$ and $120 \mathrm{mbar}$ ). Detection was performed by ultraviolet (UV) spectroscopy at a wavelength of $210 \mathrm{~nm}$. The purity of the peptides was analyzed by UV detection and electrospray mass detection using analytical reverse-phase HPLC-MS on a Nucleodur C18 HTec column $(150 \mathrm{~mm} \times 3 \mathrm{~mm}, 5 \mu \mathrm{m}$, $110 \AA$ ) and conditions identical to those of preparative HPLC. Purified peptides were lyophilized at 0.03 mbar and $-85{ }^{\circ} \mathrm{C}$ using an Alpha 2-4 LDplus Freeze-Dryer (Christ).

Antimicrobial Growth Inhibition. For all intermediate sequences, the ability to inhibit the growth of GFP-expressing Staphylococcus aureus SH1000 was measured. Minimal inhibitory concentrations (MICs) are listed in Table 1.S. aureus SH1000 expressing GFP was a gift from P. Hill (The University of Nottingham, Nottingham, U.K.) and was cultured on tryptic soy agar (Sigma-Aldrich) containing 10 $\mu \mathrm{g} \mathrm{mL}^{-1}$ chloramphenicol. To monitor bacterial growth, $1 \times$ $10^{6}$ bacteria were incubated in $200 \mu \mathrm{L}$ of nutrient broth containing $10 \mu \mathrm{g} \mathrm{mL}^{-1}$ chloramphenicol and a $50 \mu \mathrm{M}$ peptide solution (or serial dilutions thereof) in transparent Nunclon Edge 96-well plates (SIFIN GmbH). Plates were shaken for 20 $\mathrm{h}$ at $37{ }^{\circ} \mathrm{C}$ in a model M200 PRO Quad4Monochromatorbased multimode reader (Tecan, Anif, Austria). Loading the plate moats with $4 \times 3 \mathrm{~mL}$ of $0.1 \%$ agarose reduced the extent of evaporation of the culture medium. Growth was monitored 


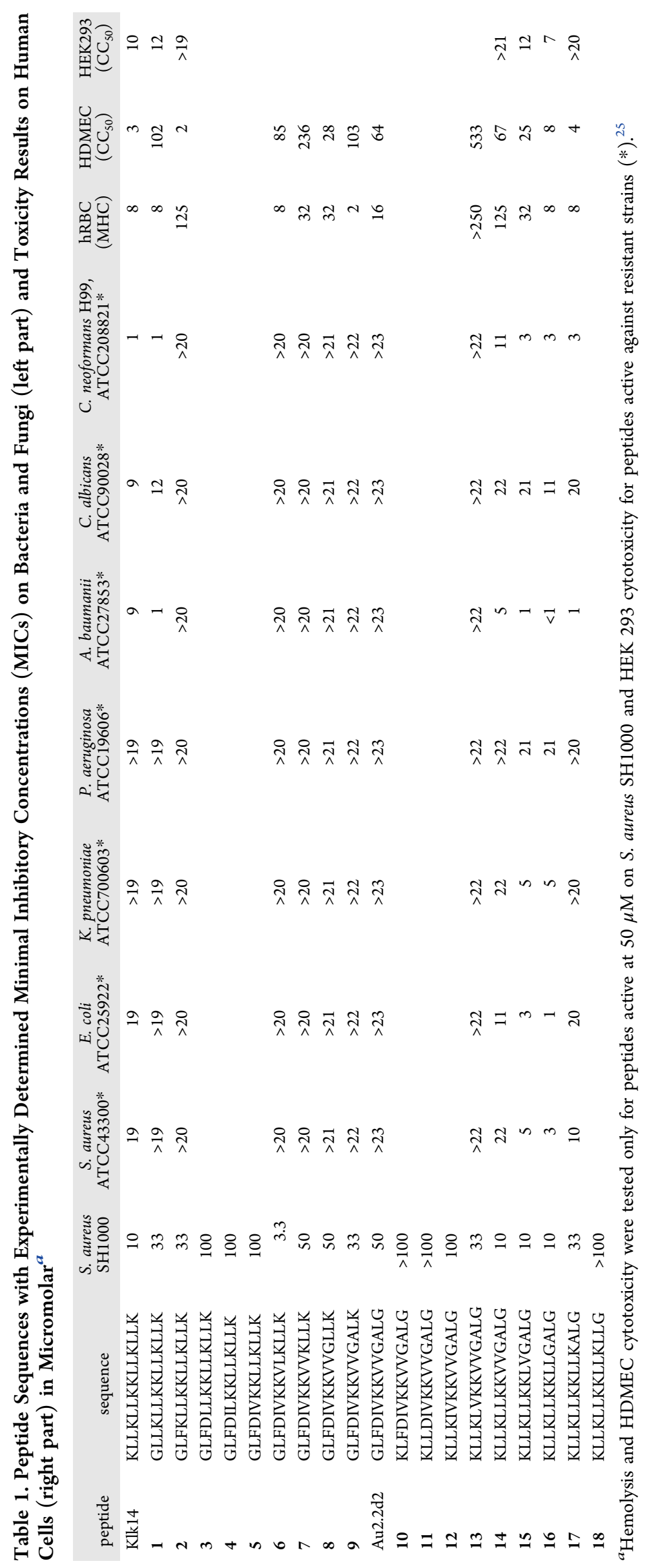


by detecting the fluorescence signal of GFP (excitation at 485 $\mathrm{nm} / 9 \mathrm{~nm}$, emission at $535 \mathrm{~nm} / 20 \mathrm{~nm}$ ) and $\mathrm{OD}_{600}$ every hour. Antimicrobial screening of the key ESKAPE bacterial pathogens S. aureus, Klebsiella pneumoniae, Acinetobacter baumannii, Pseudomonas aeruginosa, and Escherichia coli as well as the fungal pathogens Cryptococcus neoformans and Candida albicans was performed by CO-ADD (The Community for Antimicrobial Drug Discovery), funded by the Wellcome Trust (U.K.) and The University of Queensland (Australia). ${ }^{25}$ Full details of the applied methods are provided as Supporting Information.

Cytotoxicity Assay. Determination of the concentration at $50 \%$ cytotoxicity $\left(\mathrm{CC}_{50}\right)$ of human embryonic kidney cells (HEK 293) was performed by CO-ADD (The Community for Antimicrobial Drug Discovery), funded by the Wellcome Trust and The University of Queensland. ${ }^{25}$ Full details of the applied methods are provided as Supporting Information. The $\mathrm{CC}_{50}$ for HDMEC was determined using Reaction Biology Corp.'s HDMEC assay on a fee-for-service basis.

Hemolysis Assay. Fresh human blood, obtained from healthy donors (Blutspende Zürich, Zurich, Switzerland), was centrifuged for $10 \mathrm{~min}\left(800 \mathrm{~g}\right.$ and $4{ }^{\circ} \mathrm{C}$ ) to harvest the erythrocytes. These were then washed three times with phosphate-buffered saline (PBS) and resuspended in PBS to attain a final erythrocyte concentration of 5\% (v/v). Fifty microliters of the erythrocyte solution was incubated with 50 $\mu \mathrm{L}$ of a serial dilution of peptides in PBS (three technical replicates) for $1 \mathrm{~h}$ at $37{ }^{\circ} \mathrm{C}$ in a round-bottom 96-well plate (Greiner Bio-One, Frickenhausen, Germany). One hundred microliters of PBS was added to every well after incubation. After centrifugation $\left(10 \mathrm{~min}\right.$ at $800 \mathrm{~g}$ and $4{ }^{\circ} \mathrm{C}$ ), the supernatant was transferred to a flat-bottom 96-well plate (TPP AG, Trasadingen, Switzerland), and hemoglobin release was measured as the absorbance at $540 \mathrm{~nm}$ using an infinite M1000 plate reader (Tecan, Männedorf, Switzerland). Controls for zero hemolysis and 100\% hemolysis consisted of erythrocytes in PBS and 1\% Triton X-100, respectively. PBS served only as a blank reference. The percentage of hemolysis was calculated from the measured absorbance as follows:

$$
\% \text { hemolysis }=\frac{A_{\text {Peptide }}-A_{\mathrm{PBS}}}{A_{\text {Triton }}-A_{\mathrm{PBS}}} \times 100
$$

The minimal hemolytic concentration (MHC) was defined as the concentration leading to $\leq 5 \%$ hemolysis.

Linear and Circular Dichroism Spectroscopy. A Chirascan spectrometer (Applied Photophysics, Leatherhead, U.K.) was used to measure both linear dichroism (LD) and circular dichroism (CD) peptide spectra. CD was recorded using a $1 \mathrm{~mm}$ path length quartz cuvette (type 110-QS, Helma Analytics). Peptide concentrations of $30 \mu \mathrm{M}$ were measured in both water and 50\% 2,2,2-trifluoroethanol (99.8\% extra pure, Acros Organics). Near-UV CD measurements were performed in triplicate with a scanning range of $185-260 \mathrm{~nm}$ and a step size of $1 \mathrm{~nm}$ for $1 \mathrm{~s} \mathrm{~nm}^{-1}$. At every measured temperature, the sample was equilibrated for $5 \mathrm{~min}$ before the measurement was taken. With the help of Pro-Data Viewer software (Applied Photophysics, version 4.2.15), triplets were averaged, and the baseline was subtracted and smoothed (window size of 4). Analysis of CD spectra was performed with help of the modlAMP Python package. ${ }^{26}$ The helical content was calculated using the DichroWeb service portal ${ }^{27}$ by applying the CONTIN algorithm with reference set $3 .^{28} \mathrm{LD}$ spectra were recorded in the presence of large unilamellar vesicles (LUVs) with diameters of 400-500 nm, consisting of 60\% 1palmitoyl-2-oleyl-sn-glycero-3-phospho( $1^{\prime}$-rac-glycerol) (POPG) and $40 \%$ cardiolipin (CL). The chosen peptide:lipid ratio was $1: 10(100 \mu \mathrm{M}: 1 \mathrm{mM})$, and vesicle deformation for peptide alignment was achieved with a shear rate of $3960 \mathrm{~s}^{-1}$, using a spinning quartz Couette cell (Starna Scientific Ltd.). Reduced LD spectra were calculated by dividing the LD signal by the sample's absorbance under isotropic (nonspinning) conditions, as shown in eq 2.

$$
\mathrm{LD}_{\text {red }}=\frac{\mathrm{LD}}{A_{\text {iso }}}
$$

Nuclear Magnetic Resonance Spectroscopy. The secondary structures of Klk14, peptide 6, and peptide 14 were elucidated by high-resolution liquid-state NMR spectroscopy in 30\% 2,2,2-trifluoroethanol- $d_{2}$ (TFE- $d_{2}$ ). Approximately $10 \mathrm{mg}$ of peptide lyophilizate was dissolved in $350 \mu \mathrm{L}$ of $\mathrm{H}_{2} \mathrm{O}$, and the $\mathrm{pH}$ was changed to 4.5 with $1 \%$ hydrochloric acid ( $\leq 37 \%$ hydrochloric acid fuming, Sigma-Aldrich, Steinheim, Germany), aiding the complete dissolution of the sample. Finally, $150 \mu \mathrm{L}$ of TFE- $d_{2}$ (d, 98\%) (Cambridge Isotope Laboratories, Inc., Tewksbury, MA) was added, yielding a sample concentration of approximately $10 \mathrm{mM}$. Measurements were performed on a Bruker Avance III $500 \mathrm{MHz}$ spectrometer at $291 \mathrm{~K}$. Two-dimensional TOCSY (mixing time of $80 \mathrm{~ms}$ ), NOESY (mixing times of 50,100, 150, and $250 \mathrm{~ms}$ ), and double-quantum-filtered COSY were carried out using standard pulse sequences and presaturation for water suppression.

Molecular Dynamics (MD) Simulations. MD simulations over 100 ns were performed with the GROMACS molecular dynamics package, version $5.1 .2,^{29,30}$ with the AMBER99SB-ILDN force field. ${ }^{31}$ The starting structures of peptides (amidated C-terminus) were generated by projecting the three-dimensional coordinates onto an idealized $\alpha$-helix with the help of the MOE program (Molecular Operating Environment, 2015.10; Chemical Computing Group, Montreal, QC). The obtained structures were relaxed as isolated systems by energy minimization to a root-mean-square deviation (RMSD) threshold of $0.0001 \mathrm{kcal} \mathrm{mol}^{-1} \AA^{-2}$ in MOE (AMBER10:EHT force field, steepest gradient). The relaxed peptides were centered in a cubic box with periodic boundary conditions, and the box size was adjusted to obtain a minimal distance of $1 \mathrm{~nm}$ between the peptide and box border. The box was solvated (SPC/E water model), ${ }^{32}$ and $\mathrm{Cl}^{-}$or $\mathrm{Na}^{+}$ ions were added to obtain a neutral system charge. We used the SETTLE algorithm ${ }^{33}$ to keep water molecules rigid and the LINCS algorithm ${ }^{34}$ (fourth-order, one iteration) to enforce constraints of all solute bonds. The system was first subjected to energy minimization (steepest decent method, threshold of $1000 \mathrm{~kJ} \mathrm{~mol}^{-1} \mathrm{~nm}^{-1}$ ) to remove bad initial contacts. Further equilibration comprised a 50 ps simulation with leapfrog integration of the NVT ensemble with a modified Berendsen thermostat ${ }^{35}$ coupled to $300 \mathrm{~K}$ with a time step of $5 \mathrm{fs}$. This was followed by another 50 ps run with leapfrog integration of the NPT ensemble with a Parrinello-Rahman barostat ${ }^{36}$ to obtain an average system pressure of 1 bar. The Verlet cutoff scheme $^{37}$ with cutoff values of $1 \mathrm{~nm}$ was used for both shortrange electrostatic and van der Waals interactions, while particle mesh Ewald (PME) ${ }^{38}$ (grid spacing of $0.16 \mathrm{~nm}$, interpolation order of 4) accounted for long-range electrostatic interactions. The time step of the simulation was set to $2 \mathrm{fs}$ during the production runs of $100 \mathrm{~ns}$ with a leapfrog integrator. 
Secondary structure assignments were computed with the DSSP algorithm. ${ }^{39}$ A peptide's helical content was expressed as the number of residues in a helical conformation, averaged over the production run trajectory while omitting the first $5 \mathrm{~ns}$ to reduce the impact of the forced initial $\alpha$-helical peptide conformation.

Molecular Dynamics Simulations in TFE and Water. A simulation box of 125 2,2,2-trifluoroethanol molecules (parameters for AMBER99SB-ILDN according to Gerig ${ }^{40}$ and Fioroni et $\mathrm{al}^{41}$ ) and 525 water molecules (SPC/E water model), ${ }^{32}$ representing a mixture of approximately $50 \%(\mathrm{v} / \mathrm{v})$ at $20{ }^{\circ} \mathrm{C}$, was equilibrated to remove residual ordering of the solvent molecules by NVT and NPT with periodic boundary conditions for $100 \mathrm{ps}$ each. The resulting box then served as a template for solvating the peptides. After solvation, the MD simulations for TFE and water followed the same protocol as described above for the water-only systems. All calculations were performed on a cluster of 64 AMD Opteron 6376 processors.

Preparation of Giant Vesicles. Giant vesicles (GVs) that were $10-20 \mu \mathrm{m}$ in diameter were prepared by swelling dried lipid membranes as previously described. ${ }^{42}$ In brief, $1 \mathrm{~mL}$ of an aqueous solution of $1 \%(\mathrm{w} / \mathrm{v})$ agarose was added to a $250 \mathrm{~mL}$ round-bottom glass flask and distributed by slowly turning the flask. The agarose was dried under a gentle stream of nitrogen on a hot plate at $50{ }^{\circ} \mathrm{C}$. Five hundred microliters of a $1 \mu \mathrm{M}$ lipid solution, consisting of $20 \mu \mathrm{L}$ of a stock solution of POPC and a $10.8 \mu \mathrm{M}$ DiD dye solution in $536 \mu \mathrm{L}$ of a $\mathrm{MeOH} / \mathrm{CHCl}_{3}$ mixture (1:1), was then added on top of the agarose film and distributed by gentle slewing. The flask was again placed under a gentle stream of nitrogen for at least $30 \mathrm{~min}$ to remove all residual solvent. GVs were then grown by finally placing the flask in an oven at $50{ }^{\circ} \mathrm{C}$ with $4 \mathrm{~mL}$ of preheated ${ }_{d d} \mathrm{H}_{2} \mathrm{O}$ slowly added along the sidewall. After $2 \mathrm{~h}$, the swollen giant liposomes inside the residual liquid were harvested with a glass pipet. The membrane dye 1,1'-dioctadecyl-3,3,3',3'-tetramethylindodicarbocyanine perchlorate $(\mathrm{DiD})$ was obtained from Fluorochem Ltd. (Hadfield, U.K.), and 1-palmitoyl-2-oleoyl-sn-glycero-3phosphocholine (POPC) was purchased from Avanti Polar Lipids Inc. (Alabaster, AL). Three examples of GVs produced by the described procedure can be found in Figure S1. Large unilamellar vesicles (LUVs) used for LD spectroscopy were produced by the same swelling method (without dye) but subsequently extruded 21 times through a $400 \mathrm{~nm}$ pore size Whatman Cyclopore polycarbonate and polyester membrane (Sigma-Aldrich, St. Louis, MO).

Microfluidic Chip Experiments. Two-layer microfluidic chips composed of PDMS (polydimethylsiloxane, Sylgard 184) (Dow Corning, Midland, MI) were designed as described previously. ${ }^{42}$ In brief, the bottom (fluid) layer accommodates 60 microchambers with vesicle traps in the center to capture single GVs. Chambers can be closed by actuation of a valve moving down the top (pressure) layer. The chip master form was fabricated using SU-8 2015 obtained from Microchem (Newton, MA) and processed to a height of $20 \mu \mathrm{m}$ by spincoating, baking steps, exposure to a UV light source through a foil mask, development, and silanization. From this master, microfluidic chips composed of PDMS were fabricated with multilayer soft lithography and bonded to a glass slide using a plasma cleaner (PDC-32 G, Harrick, NY). To prepare chips for the experiment, ${ }_{d d} \mathrm{H}_{2} \mathrm{O}$ was pipetted into the inlet reservoirs and compressed into the chip by centrifugation at $2000 \mathrm{rpm}$ for $5 \mathrm{~min}$ to avoid air bubbles. A filtered $4 \%$ (w/v) BSA solution (Sigma-Aldrich) in PBS (Gibco Life Technologies, Paisley, U.K.) was then pumped into the chip channels by a Nanojet syringe pump $\left(10 \mu \mathrm{L} \mathrm{min}{ }^{-1}\right.$ for $10 \mathrm{~min}$, followed by a $10 \mathrm{~min}$ incubation) to coat the internal surfaces for the prevention of unspecific peptide or GV binding. Then, GVs were injected into the chip at a rate of $1-10 \mu \mathrm{L} \min ^{-1}$ for 30 min before 2 bar of pressure was applied to the donut-shaped valves in the upper layer to enclose the trapped vesicles. Afterward, the channels were flushed with $100 \mu \mathrm{M}$ peptide with $50 \mu \mathrm{M}$ calcein (Acros Organics, Morris Plains, NJ) in

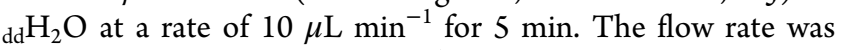

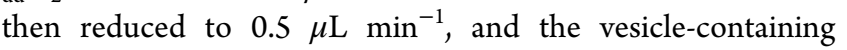
compartments were opened. Upon contact with the peptide, calcein influx and rupturing of the vesicles were monitored through microscopic imaging.

Light Microscopy. The microfluidic chips were imaged as previously described. ${ }^{42}$ Briefly, chips were mounted on the stage of an inverted microscope (IX71, Olympus) equipped with a $100 \times / 1.30 \mathrm{NA}$ oil objective. The fluorophores were excited with a SpectraX-6-LCR-SA LED system (Lumencore, Beaverton, OR). Different optical filter sets were used for imaging of (i) calcein $[474 \mathrm{~nm} / 27 \mathrm{~nm}$ BrightLine HC (excitation) and $515 \mathrm{~nm} \mathrm{HC}$ Quadband (emission)] and (ii) DiD $[635 \mathrm{~nm} / 18 \mathrm{~nm}$ BrightLine HC (excitation) and $730 \mathrm{~nm}$ HC Quadband (emission)]. In both cases, we used the dichroic mirror BrightLine Quadband BS 409/493/573/652 nm. Images of GVs were recorded with an EMCCD camera (iXon Ultra DU-897U, Andor, Belfast, Northern Ireland). For the imaging of vesicle rupture, a high-speed camera (Phantom Miro M110 camera, Vision Research) was used.

\section{RESULTS AND DISCUSSION}

The 18 designed peptides as well as the two templates were synthesized using Fmoc solid-phase peptide synthesis ${ }^{23,24}$ on a robotic synthesizer and purified by preparative LC-MS (Table S4). These linear peptides were synthesized with amidated Ctermini to increase the general membrane affinity. ${ }^{43-45}$ The two templates (Au2.2d 2 and Klk14) and 11 of the 18 designs showed growth inhibitory activity at a MIC of $\leq 50 \mu \mathrm{M}$ against the nonresistant $S$. aureus SH1000 strain (Table 1). This inhibitory activity proportion of $61 \%$ is high in comparison to those from related studies. ${ }^{46-48}$ A potential explanation for this observation could be the low MIC of the starting template Klk14, in combination with the shared pharmacophore sequence pattern of the generated peptides, allowing all of them to form distinct amphipathic helices (helical wheel plots of all peptides in Figure S10). The active peptides were further tested against five multiresistant bacteria ( $S$. aureus, E. coli, $K$. pneumoniae, $P$. aeruginosa, and A. baumannii) (see Table 1 for details on the ATCC strains) from the ESKAPE panel ${ }^{49}$ and resistant strains of the fungi $C$. albicans and C. neoformans. Five of the designs and the Klk14 template inhibited the growth of one or more resistant strains at a MIC of $\leq 22 \mu \mathrm{M}$ (Table 1 ).

The naturally occurring AMPs Aurein and Citropin contain an N-terminal GL (Gly-Leu) residue motif that is conserved in all members of this peptide family. Changing this motif led to a complete loss of activity, e.g., peptide 10 (Table 1). In comparison, changing residues 9-13 retained or even increased the activity, e.g., peptides 6-9. We therefore hypothesize that substituting residues in sequence positions with high Shannon information ${ }^{50}$ affects antimicrobial activity more than changes in less information-rich residues (Figure S9 and Tables S5 and S6). 
To be suitable for drug development, AMPs must not kill human cells. Cytotoxic concentrations leading to $50 \%$ cell death $\left(\mathrm{CC}_{50}\right)$ were determined on human dermal microvascular endothelial cells (HDMECs) and human embryonic kidney cells (HEK 293). Hemolysis of human erythrocytes (hRBCs) was determined as the minimal hemolytic concentration (MHC) leading to 5\% lysis. The results are listed in Table 1 . We identified peptide $\mathbf{1 4}$ as a selective AMP active against six resistant strains, with a calculated therapeutic index of 12.5 (fraction of the MHC for hRBC over the MIC for $S$. aureus SH1000). Peptides $\mathbf{6}$ and $\mathbf{1 5}$ also exhibited higher activity on bacteria than on human cells, albeit with calculated therapeutic indices of $<3$. The broadly active and selective peptide $\mathbf{1 4}$ is the center of the $\mathrm{C}$ - to $\mathrm{N}$-terminal morphing series (Figure 1), incorporating the cationic N-terminal half of Klk14 and the hydrophobic C-terminal portion of Au2.2d2. Sequences 14-16 reveal that the $\mathrm{Val}^{9} \rightarrow \mathrm{Leu}^{9}$ and $\mathrm{Val}^{10} \rightarrow$ $\mathrm{Leu}^{10}$ transitions led to a pronounced increase in membranolytic activity against both resistant strains and human cells. Val and Leu side chains share the same pharmacophore, have similar hydrophobicity values, and are generally considered similar. ${ }^{51}$ One explanation for this steep activity increase might thus be found in the different helix-stabilizing properties of these two residues. ${ }^{52}$

For verification of $\alpha$-helical structure, we performed CD spectroscopy of all peptides in both water and a 50\% TFE/ water mixture $(\mathrm{v} / \mathrm{v})$ at room temperature (Figure 2). The

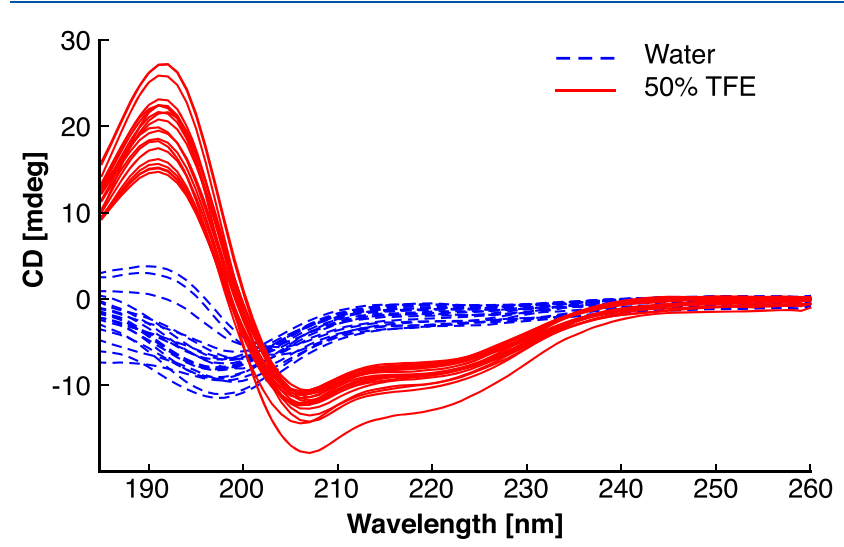

Figure 2. Circular dichroism spectra of all peptides. All measurements were performed under equal conditions with a peptide concentration of $33 \mu \mathrm{M}$. The maximum at $190 \mathrm{~nm}$ and the minima at 208 and 222 $\mathrm{nm}$ are characteristic of $\alpha$-helical secondary structures. Every line represents the average of three technical replicates under the indicated condition (blue, water; red, 50\% TFE in water).

templates and all designs displayed characteristic spectra of $\alpha$ helices in 50\% TFE, whereas all spectra in pure water suggested random coil structures. This observation corroborates our design hypothesis of the intermediate sequences adopting $\alpha$-helical structures in a hydrophobic environment (50\% TFE).

Because these CD spectra did not explain the observed differences in peptide activity and selectivity, we hypothesized that the structural stability of the $\alpha$-helices could be a selectivity-determining factor. Therefore, we recorded $\mathrm{CD}$ at increasing temperatures in a 50\% TFE/water mixture (v/v), using the $\alpha$-helical stability at elevated temperatures as a surrogate for structural flexibility. The changes in the CD signal at three typical $\alpha$-helical wavelengths $(192,208$, and 222 $\mathrm{nm})$ between the lowest $\left(30{ }^{\circ} \mathrm{C}\right)$ and highest $\left(75^{\circ} \mathrm{C}\right)$ temperature were compared to the activity and selectivity of all peptides that inhibited S. aureus SH1000 (Table 2 and

Table 2. Correlation Analysis of Peptide Activity with Relative Differences in CD Spectra at Increasing Temperatures $^{a}$

$\begin{array}{lccc} & \text { S. aureus MIC } & \text { HDMEC CC }_{50} & \text { hRBC MHC } \\ \partial 192 \mathrm{~nm} & 0.082 & 0.608 & 0.773 \\ \partial 208 \mathrm{~nm} & 0.038 & 0.609 & 0.770 \\ \partial 222 \mathrm{~nm} & 0.096 & 0.537 & 0.739\end{array}$

${ }^{a}$ Signal differences $(\partial)$ at three characteristic wavelengths comparing spectra recorded at $30-75{ }^{\circ} \mathrm{C}$ were correlated with peptide minimal inhibitory concentrations (MICs) on S. aureus SH1000, cytotoxicity on human dermal microvascular epithelial cells $\left(\mathrm{HDMEC} \mathrm{CC}_{50}\right)$, and the minimal hemolytic concentration of human red blood cells (hRBC MHC). Numbers are Pearson product-moment correlation coefficients ranging from 0 (no correlation) to 1 (perfect correlation).

Figure S6). The chosen temperatures do not have a physiological meaning but were selected for the sole purpose of determining $\alpha$-helical stability. We observed that increasing relative differences in $\mathrm{CD}$ spectra recorded at low and high temperatures suggest greater peptide selectivity (comparing $S$. aureus SH1000 vs HDMEC and hRBC). This result points to increased structural flexibility at elevated temperatures as a discriminative feature of cell-type selective AMPs and potentially explains why the $\mathrm{Val} \rightarrow$ Leu modification impacts AMP selectivity. Measuring CD at high (although physiologically nonrelevant) temperatures may be a suitable method for determining AMP selectivity.

Hydrophobicity has been suggested to be critically important for the activity of model peptides against Grampositive bacteria. ${ }^{53,54}$ In contrast, peptides 13-16 have the same global Eisenberg hydrophobicity value of 0.23 and a hydrophobic moment of 0.61 but differ in their selectivity (Table 1). However, the calculated global flexibility (modlAMP flexibility scale ${ }^{26}$ ) of peptides $13-17$ is correlated to their hemolytic activity (Pearson $r^{2}=0.74 ; p$ value for noncorrelation of 0.16 ), with the more flexible peptides (peptides 13-15) being less hemolytic, but apparently does not correlate with the peptides' antimicrobial activity (Table 3).

To further investigate structural flexibility and learn about the potential mechanism of action of the peptides, we performed $\mathrm{MD}$ simulations for all peptides in water and a $50 \% \mathrm{TFE} /$ water mixture (v/v) over $100 \mathrm{~ns}$. MD confirmed the CD measurements in that all peptides adopted helical

Table 3. Comparison of the Global Flexibility [modlAMP flexibility scale ${ }^{26}$ (Table S7)] to the Hemolytic Activity (MHC) of Peptides 13-17 ${ }^{a}$

$\begin{array}{ccc}\text { peptide } & \text { MHC }(\mu \mathrm{M}) & \text { flexibility } \\ 13 & >250 & 0.527 \\ 14 & 125 & 0.521 \\ 15 & 32 & 0.515 \\ 16 & 8 & 0.509 \\ 17 & 8 & 0.488\end{array}$

${ }^{a}$ A peptide's flexibility is calculated by averaging the flexibility values of the individual amino acids. The flexibility scale ranges from 0 to 1 (see Table S7 for individual amino acids). 
structures in the TFE simulations, whereas they unfolded during simulations in pure water. The designs incorporating the C-terminus of Au2.2d 2 seemed to partially unfold over time [i.e., peptide 14 (Figure S3)]. In contrast, the peptides containing the N-terminus of Au2.2d 2 did not show this behavior [i.e., peptide 4 (Figure S2)]. A simulation snapshot of peptide 14 shows its C-terminus "snorkeling" into the TFE layer, suggesting that the designed peptides enter membranes by the adoption of oblique oriented $\alpha$-helices (Figure 3 ). This

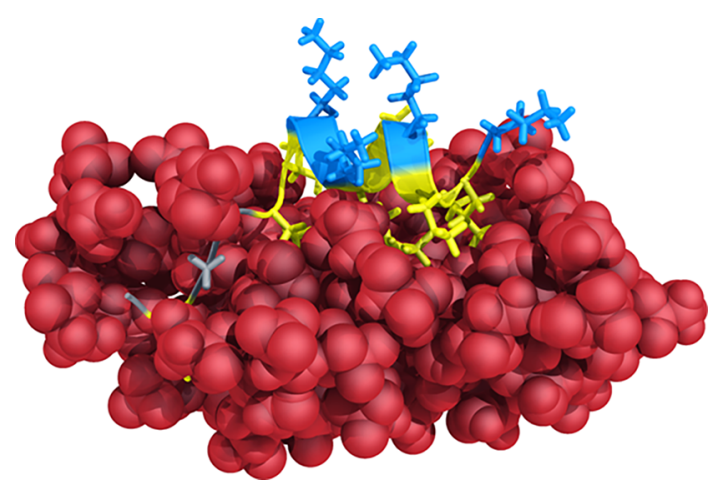

Figure 3. Molecular dynamics simulation snapshot of peptide 14 with its C-terminal end (left) snorkeling into the TFE layer (red spheres). This observation supports the hypothesis of membrane invasion by the adoption of oblique oriented $\alpha$-helices.

mechanism of membrane invasion by amphibian AMPs has been previously described. ${ }^{55}$ Profile plots of hydrophobicity and hydrophobic moments can also hint at oblique orientation. Figure 4 reveals the increase in hydrophobicity toward the C-

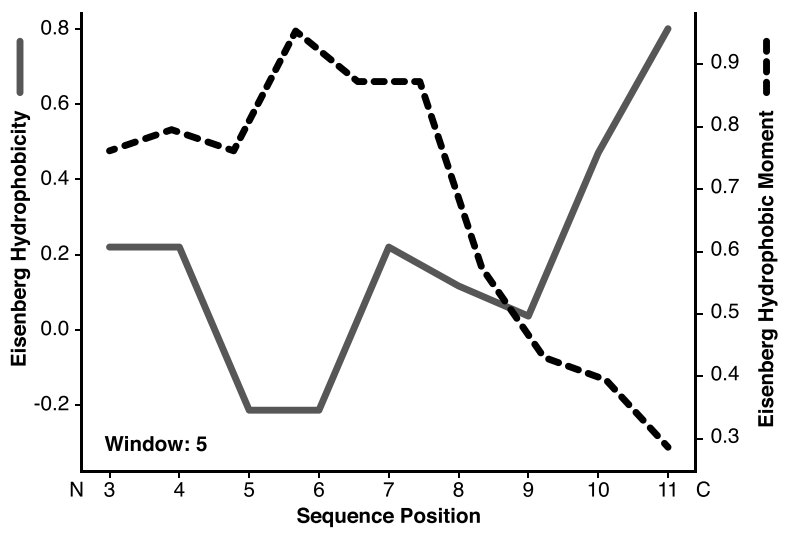

Figure 4. Hydropathy plot of peptide 14. The gray line with the corresponding left $y$-axis shows the Eisenberg hydrophobicity, and the dashed black line with the corresponding right $y$-axis shows the calculated hydrophobic moment. The linear hydrophobicity gradient increases toward the $\mathrm{C}$-terminus. The results suggest that the more flexible hydrophobic C-terminal part initiates membrane insertion.

terminus of peptide $\mathbf{1 4}$ and the concurrent decrease in the helical hydrophobic moment, suggesting an unstructured hydrophobic tail that can penetrate a lipid bilayer. These calculations support the observed peptide "snorkeling" in the MD simulations.

The kinetics and mechanism of action were further investigated using giant vesicles (diameters of $10-20 \mu \mathrm{m}$ ) in a microfluidic chamber. As the $\alpha$-helices of all our sequences are too short (14 residues) to span lipid bilayers, the formation of barrel-stave pores is impossible. Video sequences recorded for peptide 6 (the most active peptide on S. aureus SH1000) imply a fast, carpet-forming membranolytic event ${ }^{55}$ upon the accumulation of peptide molecules on the vesicle surface (Figure 5, Video S1, and Video S2). Consequently, LD

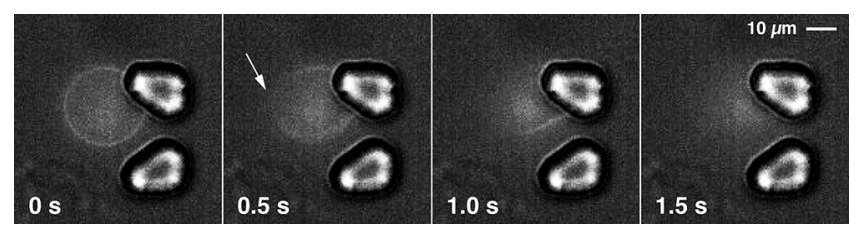

Figure 5. Snapshots of a video sequence of a giant POPC vesicle (diameter of $20 \mu \mathrm{m}$ ) with membrane-incorporated DiD dye, floating in the microfluidic chip chamber in the presence of $50 \mu \mathrm{M}$ peptide 6 , the most active peptide on $S$. aureus $\mathrm{SH} 1000$. The flow was from left to right at a rate of $0.5 \mu \mathrm{L} \mathrm{min}{ }^{-1}$. Two micropillars acted as a hydrodynamic trap for the vesicle. The white arrow indicates the position at which the lipid membrane of the vesicle started to rupture upon exposure to peptide 6 .

spectroscopy was performed to obtain preliminary information about the relative orientation of templates Au2.2d 2 and Klk14, as well as peptides $\mathbf{6}$ and $\mathbf{1 4}$ in model membranes. The LD spectra obtained showed marked differences between Au2.2d 2 and the three other peptides, with 6 and 14 resembling the Klk14 template (Figure S7). The spectrum of Au2.2d2 is clearly distinct from the LD spectra of the other three peptides. However, these findings cannot be clearly related to the observed differences in growth inhibitory or cytotoxic activity, or to the recorded $\mathrm{CD}$ spectra.

To verify the observed relationship between peptide flexibility and cell selectivity and potentially illuminate differences in LD spectra, we performed solution NMR spectroscopy with Klk14, as well as peptides 6 and 14, in a $30 \% \mathrm{TFE} /$ water mixture. The structure of $\mathrm{Au} 2.2 \mathrm{~d} 2$ in a $30 \%$ TFE/water mixture was already known from a previous study. ${ }^{14}$ Structural ensembles of the 10 lowest-energy structures of these four peptides are shown in Figure 6 . Terminal flexibility observed for peptide 14 in MD simulations could partially be confirmed by NMR. Compared to peptide 6 , the C-terminus of peptide $\mathbf{1 4}$ is indeed more flexible, confirmed by the respective Ramachandran plots (Figure S8). Overall, Klk14 shows the fewest nuclear Overhauser effect (NOE)-derived NMR constraints in this measurement. A decrease in the number of observed NOEs together with line broadening is typical for increased exchange phenomena due to flexibility. One can observe such a drop in the number of residual constraints for the terminal, hydrophobic portion (sequence of GALG) of peptide 14 relative to its central part and to the corresponding residues of peptide Klk14. Both the $\mathrm{MD}$ snapshot shown in Figure 3 and the MD trajectory of peptide 14 simulated in TFE (Figure S2) are in agreement with the NMR result. These findings provide a potential explanation for the different orientation of $\mathrm{Au} 2.2 \mathrm{~d} 2$ in contact with lipid vesicles (which was observed during the LD experiment), as this peptide was previously found to have higher helical stability in terms of NMR constraints. ${ }^{14}$ With regard to the differences in TFE percentage between the experimental NMR conditions (30\% TFE) and the MD simulations (50\% TFE), studies by Fioroni et al. have shown that secondary structure-stabilizing TFE cluster formation 
a)
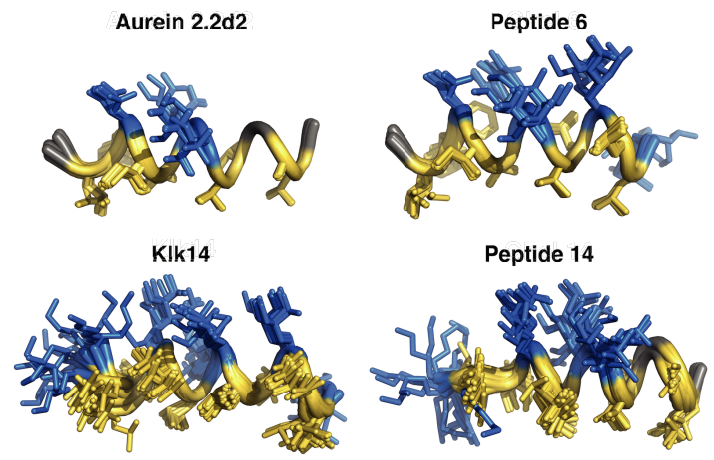

b) KIk14
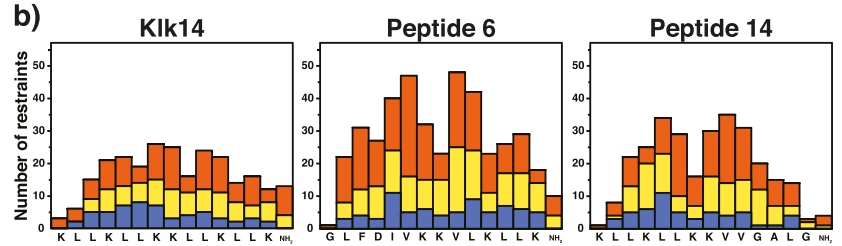

Figure 6. Solution NMR results. (a) Structural ensemble of the 10 lowest-energy structures of templates Au2.2d2 (PDB entry 5MXL) and Klk14 (PDB entry 6HNG), as well as of peptide 6 (PDB entry $6 \mathrm{HNE}$ ) and peptide 14 (PDB entry 6HNH). Hydrophobic residues are colored yellow, and polar ones blue. Gly is colored gray. (b) Nuclear Overhauser effect (NOE) statistics plotted against the corresponding residues. Blue bars represent intraresidue NOEs, yellow bars sequential restraints, and orange bars medium-range NOEs $(1<|i-j|<5)$. Restraints for Au2.2d2 can be found in ref 15 . Structures were modeled from spectra recorded in a $30 \%$ TFE/water mixture.

reaches a maximum at approximately 30\% TFE and water, obviating large differences relative to that in $50 \%$ TFE used in the MD simulation. ${ }^{41}$

In summary, systematic "morphing" of the natural helical AMP Aurein Au2.2d2 into the synthetic helical AMP Klk14 resulted in chimeric sequences exhibiting general antimicrobial activity and selectivity against several antibiotic-resistant bacteria and fungi. Helical amphipathicity turned out to be a necessary but insufficient condition for the membranolytic activity of these designs. For the new AMPs that kill $S$. aureus SH1000, we detected a positive correlation between the peptides' structural flexibility at elevated temperatures and their cell-type selectivity. In agreement with the results from biophysical measurements, MD simulations suggested that active peptides with higher selectivity toward bacteria possess a greater proportion of flexible areas than less selective AMPs. The NMR structures obtained for peptides 6 and 14 additionally confirmed the more pronounced flexibility of the C-terminal part of the more selective peptide 14. Together, these results support the working hypothesis of partial structural flexibility playing a key role in the cell-type selectivity of AMPs.

Single changes of certain amino acids led to a loss of antibacterial activity, even when the pharmacophore features of the original and the substituted residues were preserved. With current AMP prediction models being unable to forecast such steep activity cliffs, the systematic transformation approach presented here offers a suitable, template-based optimization strategy for helical AMPs.

The results of this study can be linked to previous work. For example, Cheng et al. investigated the relative importance of a hydrophobic residue in position 13 of Aurein 2.2 for peptide structure and membranolytic activity. ${ }^{10}$ Accordingly, the exact nature of the residue appears to be less important, as long as it is hydrophobic. To better distinguish between amino acids of the same pharmacophore type, Senes et al. developed an empirical potential of insertion of protein into lipid bilayers (Ez). ${ }^{56}$ The Ez potential determines the free energy profile for each residue of the peptide sequence as a continuous function of the depth of insertion into the lipid membrane. Global potential can then be determined via a reverse Boltzmann statistical approach to convert propensities into pseudoenergies of membrane insertion. Giguère et al. presented learning algorithms with generic string kernels to capture patterns responsible for peptide bioactivity. ${ }^{57}$ A de novodesigned sequence (WWKRWKKLRRIFLML) exhibited MICs of $4 \mu \mathrm{M}$ against both E. coli and S. aureus. ${ }^{58}$ Combining the calculated sequence motifs by Giguère et al., the preferred location and membrane depth propensities of Trp residues in AMPs, ${ }^{57,59}$ and the results of the study presented here, one could propose a "universal" membranolytic sequence. Accordingly, such a minimalist helical AMP sequence would need to have one or several anchoring Trp or Tyr residues in the Nterminal part, repetitive cationic amino acids to form an amphipathic secondary structure, and a hydrophobic Cterminus to, at least initially, interact with the hydrophobic core of the membrane. In fact, it has been suggested that a majority of $\alpha$-helical AMPs are candidates for obliquely oriented helix formation. ${ }^{60}$ For several AMPs (Aurein 1.2, Citropin 1.1, Maculatin 1.1, and Carein 1.1), such an oblique membrane insertion at an angle of approximately $50^{\circ}$ was demonstrated by solid-state NMR and oriented CD spectroscopy. ${ }^{59}$ Taken together, the findings presented here should foster future AMP design studies toward more selective and highly active $\alpha$-helical AMPs, based on partial peptide flexibility as a driving factor.

\section{ASSOCIATED CONTENT}

\section{SI Supporting Information}

The Supporting Information is available free of charge at https://pubs.acs.org/doi/10.1021/acs.biochem.0c00565.

Supporting data and methodological details about the computational activity predictions, molecular dynamics simulations, CD spectroscopy, solution-phase NMR, peptide flexibility analysis, and $\mathrm{CO}-\mathrm{ADD}$ antimicrobial and antifungal screening (PDF)

Video sequence (version 1) of the interaction of peptide 6 with a giant POPC vesicle (AVI)

Video sequence (version 2) of the interaction of peptide 6 with a giant POPC vesicle (AVI)

\section{AUTHOR INFORMATION}

\section{Corresponding Author}

Gisbert Schneider - Department of Chemistry and Applied Biosciences, ETH Zurich, 8093 Zürich, Switzerland; ○ orcid.org/0000-0001-6706-1084; Email: gisbert@ethz.ch

\section{Authors}

Alex T. Müller - Department of Chemistry and Applied Biosciences, ETH Zurich, 8093 Zürich, Switzerland

Gernot Posselt - Department of Biosciences, Division of Microbiology, Paris Lodron University of Salzburg, 5020 Salzburg, Austria 
Gisela Gabernet - Department of Chemistry and Applied Biosciences, ETH Zurich, 8093 Zürich, Switzerland

Claudia Neuhaus - Department of Chemistry and Applied Biosciences, ETH Zurich, 8093 Zürich, Switzerland

Simon Bachler - Department of Biosystems Science and Engineering, ETH Zurich, 4058 Basel, Switzerland

Markus Blatter - Novartis Institutes for BioMedical Research, Novartis Pharma AG, 4002 Basel, Switzerland

Bernhard Pfeiffer - Department of Chemistry and Applied Biosciences, ETH Zurich, 8093 Zürich, Switzerland

Jan A. Hiss - Department of Chemistry and Applied Biosciences, ETH Zurich, 8093 Zürich, Switzerland

Petra S. Dittrich - Department of Biosystems Science and Engineering, ETH Zurich, 4058 Basel, Switzerland; (1) orcid.org/0000-0001-5359-8403

Karl-Heinz Altmann - Department of Chemistry and Applied Biosciences, ETH Zurich, 8093 Zürich, Switzerland; 이이이.org/0000-0002-0747-9734

Silja Wessler - Department of Biosciences, Division of Microbiology, Paris Lodron University of Salzburg, 5020 Salzburg, Austria

Complete contact information is available at: https://pubs.acs.org/10.1021/acs.biochem.0c00565

\section{Author Contributions}

A.T.M. designed peptide sequences. A.T.M., G.G., and C.N. performed peptide syntheses. A.T.M. and G.P. performed activity assays. A.T.M. and S.B. performed microfluidics assays. A.T.M. ran molecular dynamics simulations. B.P. recorded NMR spectra. M.B. performed NMR analysis. J.A.H., P.S.D., K.-H.A., S.W., and G.S. designed and supervised the study. A.T.M. and G.S. wrote the manuscript.

\section{Funding}

This research was supported by ETH Research Grant ETH-01 12-1 (to G.S.), the Swiss National Science Foundation (Grant 200021 157190 to G.S. and J.A.H. and Grant CRSII2_160699 to G.S.), and the Austrian Science Fund (FWF Grants P_24074 and P_24315 to S.W.).

\section{Notes}

The authors declare the following competing financial interest(s): G.S. is a co-founder of inSili.com LLC, Zurich, Switzerland, and M.B. is a paid employee of Novartis AG, Basel, Switzerland.

\section{ACKNOWLEDGMENTS}

The authors thank S. Haller, P. Rabatscher, and J. A. Fuchs for technical support.

\section{REFERENCES}

(1) World Health Organization (2015) Worldwide Country Situation Analysis: Response to Antimicrobial Resistance. Geneva.

(2) Fjell, C. D., Hiss, J. A., Hancock, R. E. W., and Schneider, G. (2012) Designing antimicrobial peptides: form follows function. Nat. Rev. Drug Discovery 11 (1), 37-51.

(3) da Cunha, N. B., Cobacho, N. B., Viana, J. F. C., Lima, L. A., Sampaio, K. B. O., Dohms, S. S. M., Ferreira, A. C. R., de la FuenteNúñez, C., Costa, F. F., Franco, O. L., and Dias, S. C. (2017) The next generation of antimicrobial peptides (AMPs) as molecular therapeutic tools for the treatment of diseases with social and economic impacts. Drug Discovery Today 22 (2), 234-248.

(4) Gabernet, G., Müller, A. T., Hiss, J. A., and Schneider, G. (2016) Membranolytic anticancer peptides. MedChem Comm 7 (12), 22322245.
(5) Rios, A. C., Moutinho, C. G., Pinto, F. C., Del Fiol, F. S., Jozala, A., Chaud, M. V., Vila, M. M. D. C., Teixeira, J. A., and Balcão, V. M. (2016) Alternatives to overcoming bacterial resistances: state-of-theart. Microbiol. Res. 191, 51-80.

(6) Wang, G., Li, X., and Wang, Z. (2016) APD3: the antimicrobial peptide database as a tool for research and education. Nucleic Acids Res. 44 (D1), D1087-D1093.

(7) Waghu, F. H., Gopi, L., Barai, R. S., Ramteke, P., Nizami, B., and Idicula-Thomas, S. (2014) CAMP: Collection of sequences and structures of antimicrobial peptides. Nucleic Acids Res. 42 (D1), D1154-D1158.

(8) Aguilera-Mendoza, L., Marrero-Ponce, Y., Tellez-Ibarra, R., Llorente-Quesada, M. T., Salgado, J., Barigye, S. J., and Liu, J. (2015) Overlap and diversity in antimicrobial peptide databases: Compiling a non-redundant set of sequences. Bioinformatics 31 (15), 2553-2559.

(9) Schneider, G., Lin, Y.-C., Koch, C. P., Pillong, M., Perna, A. M., Reutlinger, M., and Hiss, J. A. (2013) Adaptive peptide design. Chimia 67 (12), 859-863.

(10) Solstad, R. G., Johansen, C., Stensvåg, K., Strøm, M. B., and Haug, T. (2020) Structure-activity relationship studies of shortened analogues of the antimicrobial peptide EeCentrocin 1 from the sea urchin Echinus esculentus. J. Pept. Sci. 26 (2), e3233.

(11) Cheng, J. T. J., Hale, J. D., Kindrachuk, J., Jessen, H., Elliott, M., Hancock, R. E. W., and Straus, S. K. (2010) Importance of residue 13 and the C-terminus for the structure and activity of the antimicrobial peptide aurein 2.2. Biophys. J. 99 (9), 2926-2935.

(12) Strøm, M. B., Haug, B. E., Skar, M. L., Stensen, W., Stiberg, T., and Svendsen, J. S. (2003) The pharmacophore of short cationic antibacterial peptides. J. Med. Chem. 46 (9), 1567-1570.

(13) Rozek, T., Wegener, K. L., Bowie, J. H., Olver, I. N., Carver, J. A., Wallace, J. C., and Tyler, M. J. (2000) The antibiotic and anticancer active aurein peptides from the Australian bell frogs Litoria aurea and Litoria raniformis. Eur. J. Biochem. 267 (17), 5330-5341.

(14) Wegener, K. L., Wabnitz, P. A., Carver, J. A., Bowie, J. H., Chia, B. C. S., Wallace, J. C., and Tyler, M. J. (1999) Host defence peptides from the skin glands of the Australian Blue Mountains tree-frog Litoria citropa. Solution structure of the antibacterial peptide citropin 1.1. Eur. J. Biochem. 265 (2), 627-637.

(15) Stutz, K., Müller, A. T., Hiss, J. A., Schneider, P., Blatter, M., Pfeiffer, B., Posselt, G., Kanfer, G., Kornmann, B., Wrede, P., Altmann, K.-H., Wessler, S., and Schneider, G. (2017) Peptide-membrane interaction between targeting and lysis. ACS Chem. Biol. 12 (9), 2254-2259.

(16) Bertocco, A., Formaggio, F., Toniolo, C., Broxterman, Q. B., Epand, R. F., and Epand, R. M. (2003) Design and function of a conformationally restricted analog of the influenza virus fusion peptide. J. Pept. Res. 62 (1), 19-26.

(17) Babii, O., Afonin, S., Schober, T., Komarov, I. V., and Ulrich, A. S. (2017) Flexibility vs rigidity of amphipathic peptide conjugates when interacting with lipid bilayers. Biochim. Biophys. Acta, Biomembr. 1859 (12), 2505-2515.

(18) Berman, H. M., Westbrook, J., Feng, Z., Gilliland, G., Bhat, T. N., Weissig, H., Shindyalov, I. N., and Bourne, P. E. (2000) The Protein Data Bank. Nucleic Acids Res. 28 (1), 235-242.

(19) Sun, J., Xia, Y., Li, D., Du, Q., and Liang, D. (2014) Relationship between peptide structure and antimicrobial activity as studied by de novo designed peptides. Biochim. Biophys. Acta, Biomembr. 1838 (12), 2985-2993.

(20) Han, H. M., Gopal, R., and Park, Y. (2016) Design and membrane-disruption mechanism of charge-enriched AMPs exhibiting cell selectivity, high-salt resistance, and anti-biofilm properties. Amino Acids 48 (2), 505-522.

(21) Migliolo, L., Felício, M. R., Cardoso, M. H., Silva, O. N., Xavier, M.-A. E., Nolasco, D. O., de Oliveira, A. S., Roca-Subira, I., Vila Estape, J., Teixeira, L. D., Freitas, S. M., Otero-Gonzalez, A. J., Gonçalves, S., Santos, N. C., and Franco, O. L. (2016) Structural and functional evaluation of the palindromic alanine-rich antimicrobial peptide Pa-MAP2. Biochim. Biophys. Acta, Biomembr. 1858 (7), 14881498. 
(22) Abdel Monaim, S. A. H., Ramchuran, E. J., El-Faham, A., Albericio, F., and de la Torre, B. G. (2017) Converting teixobactin into a cationic antimicrobial peptide (AMP). J. Med. Chem. 60 (17), $7476-7482$.

(23) Merrifield, R. B. (1963) Solid phase peptide synthesis. I. The synthesis of a tetrapeptide. J. Am. Chem. Soc. 85 (14), 2149-2154.

(24) Chan, W. C., and White, P. D. (2000) Fmoc Solid Phase Peptide Synthesis, Oxford University Press, Oxford, U.K.

(25) Blaskovich, M. A. T., Zuegg, J., Elliott, A. G., and Cooper, M. A. (2015) Helping chemists discover new antibiotics. ACS Infect. Dis. 1 (7), 285-287.

(26) Müller, A. T., Gabernet, G., Hiss, J. A., and Schneider, G. (2017) modlAMP: Python for antimicrobial peptides. Bioinformatics 33 (17), 2753-2755.

(27) Whitmore, L., and Wallace, B. A. (2008) Protein secondary structure analyses from circular dichroism spectroscopy: Methods and reference databases. Biopolymers 89 (5), 392-400.

(28) Greenfield, N. J. (2006) Using circular dichroism spectra to estimate protein secondary structure. Nat. Protoc. 1 (6), 2876-2890.

(29) Berendsen, H. J. C., van der Spoel, D., and van Drunen, R. (1995) GROMACS: a message-passing parallel molecular dynamics implementation. Comput. Phys. Commun. 91 (1-3), 43-56.

(30) Abraham, M. J., Murtola, T., Schulz, R., Páll, S., Smith, J. C., Hess, B., and Lindahl, E. (2015) GROMACS: high performance molecular simulations through multi-level parallelism from laptops to supercomputers. SoftwareX 1-2, 19-25.

(31) Best, R. B., and Hummer, G. (2009) Optimized molecular dynamics force fields applied to the helix-coil transition of polypeptides. J. Phys. Chem. B 113 (26), 9004-9015.

(32) Berendsen, H. J. C., Grigera, J. R., and Straatsma, T. P. (1987) The missing term in effective pair potentials. J. Phys. Chem. 91 (24), 6269-6271.

(33) Miyamoto, S., and Kollman, P. A. (1992) Settle: an analytical version of the SHAKE and RATTLE algorithm for rigid water models. J. Comput. Chem. 13 (8), 952-962.

(34) Hess, B., Bekker, H., Berendsen, H. J. C., and Fraaije, J. G. E. M. (1997) LINCS: a linear constraint solver for molecular simulations. J. Comput. Chem. 18 (12), 1463-1472.

(35) Berendsen, H. J. C., Postma, J. P. M., van Gunsteren, W. F., DiNola, A., and Haak, J. R. (1984) Molecular dynamics with coupling to an external bath. J. Chem. Phys. 81 (8), 3684.

(36) Parrinello, M., and Rahman, A. (1981) Polymorphic transitions in single crystals: a new molecular dynamics method. J. Appl. Phys. 52 (12), 7182.

(37) Verlet, L. (1968) Computer "experiments" on classical fluids. II. Equilibrium correlation functions. Phys. Rev. 165 (1), 201-214.

(38) Essmann, U., Perera, L., Berkowitz, M. L., Darden, T., Lee, H., and Pedersen, L. G. (1995) A smooth particle mesh Ewald method. J. Chem. Phys. 103 (19), 8577.

(39) Kabsch, W., and Sander, C. (1983) Dictionary of protein secondary structure: pattern recognition of hydrogen-bonded and geometrical features. Biopolymers 22 (12), 2577-2637.

(40) Gerig, J. T. (2015) Further efforts toward a molecular dynamics force field for simulations of peptides in $40 \%$ trifluoroethanol-water. J. Phys. Chem. B 119 (16), 5163-5175.

(41) Fioroni, M., Burger, K., Mark, A. E., and Roccatano, D. (2000) A new 2,2,2-trifluoroethanol model for molecular dynamics simulations. J. Phys. Chem. B 104 (51), 12347-12354.

(42) Pillong, M., Hiss, J. A., Schneider, P., Lin, Y.-C., Posselt, G., Pfeiffer, B., Blatter, M., Müller, A. T., Bachler, S., Neuhaus, C. S., Dittrich, P. S., Altmann, K.-H., Wessler, S., and Schneider, G. (2017) Rational design of membrane-pore-forming peptides. Small 13 (40), 1701316.

(43) Strandberg, E., Tiltak, D., Ieronimo, M., Kanithasen, N., Wadhwani, P., and Ulrich, A. S. (2007) Influence of C-terminal amidation on the antimicrobial and hemolytic activities of cationic $\alpha$ helical peptides. Pure Appl. Chem. 79 (4), 717-728.
(44) Zhu, S., Aumelas, A., and Gao, B. (2011) Convergent evolution-guided design of antimicrobial peptides derived from influenza A virus hemagglutinin. J. Med. Chem. 54 (4), 1091-1095.

(45) Dennison, S. R., and Phoenix, D. A. (2011) Influence of Cterminal amidation on the efficacy of modelin-5. Biochemistry 50 (9), $1514-1523$.

(46) Khara, J. S., Lim, F. K., Wang, Y., Ke, X.-Y., Voo, Z. X., Yang, Y. Y., Lakshminarayanan, R., and Ee, P. L. R. (2015) Designing $\alpha$-helical peptides with enhanced synergism and selectivity against Mycobacterium smegmatis: discerning the role of hydrophobicity and helicity. Acta Biomater. 28, 99-108.

(47) Kim, H., Jang, J. H., Kim, S. C., and Cho, J. H. (2014) De novo generation of short antimicrobial peptides with enhanced stability and cell specificity. J. Antimicrob. Chemother. 69 (1), 121-132.

(48) Kang, S. J., Won, H. S., Choi, W. S., and Lee, B. J. (2009) De novo generation of antimicrobial LK peptides with a single tryptophan at the critical amphipathic interface. J. Pept. Sci. 15 (9), 583-588.

(49) Boucher, H. W., Talbot, G. H., Bradley, J. S., Edwards, J. E., Gilbert, D., Rice, L. B., Scheld, M., Spellberg, B., and Bartlett, J. (2009) Bad bugs, no drugs: no ESKAPE! An update from the Infectious Diseases Society of America. Clin. Infect. Dis. 48 (1), 1-12.

(50) Shannon, C. E. (1948) A mathematical theory of communication. Bell Syst. Tech. J. 27 (3), 379-423.

(51) Lyu, P. C., Sherman, J. C., Chen, A., and Kallenbach, N. R. (1991) Alpha-helix stabilization by natural and unnatural amino acids with alkyl side chains. Proc. Natl. Acad. Sci. U. S. A. 88 (12), 53175320.

(52) Schmidtchen, A., Pasupuleti, M., and Malmsten, M. (2014) Effect of hydrophobic modifications in antimicrobial peptides. Adv. Colloid Interface Sci. 205, 265-274.

(53) Dathe, M., Wieprecht, T., Nikolenko, H., Handel, L., Maloy, W. L., MacDonald, D. L., Beyermann, M., and Bienert, M. (1997) Hydrophobicity, hydrophobic moment and angle subtended by charged residues modulate antibacterial and haemolytic activity of amphipathic helical peptides. FEBS Lett. 403 (2), 208-212.

(54) Bhaskaran, R., and Ponnuswamy, P. K. (1988) Positional flexibilities of amino acid residues in globular proteins. Int. J. Pept. Protein Res. 32 (4), 241-255.

(55) Brogden, K. A. (2005) Antimicrobial peptides: pore formers or metabolic inhibitors in bacteria? Nat. Rev. Microbiol. 3 (3), 238-250.

(56) Senes, A., Chadi, D. C., Law, P. B., Walters, R. F. S., Nanda, V., and DeGrado, W. F. (2007) Ez, a depth-dependent potential for assessing the energies of insertion of amino acid side-chains into membranes: derivation and applications to determining the orientation of transmembrane and interfacial helices. J. Mol. Biol. 366 (2), 436-448.

(57) Giguère, S., Laviolette, F., Marchand, M., Tremblay, D., Moineau, S., Liang, X., Biron, É., and Corbeil, J. (2015) Machine learning assisted design of highly active peptides for drug discovery. PLoS Comput. Biol. 11 (4), e1004074.

(58) Fimland, G., Eijsink, V. G. H., and Nissen-Meyer, J. (2002) Mutational analysis of the role of tryptophan residues in an antimicrobial peptide. Biochemistry 41 (30), 9508-9515.

(59) Marcotte, I., Wegener, K. L., Lam, Y.-H., Chia, B. C. S., de Planque, M. R. R., Bowie, J. H., Auger, M., and Separovic, F. (2003) Interaction of antimicrobial peptides from Australian amphibians with lipid membranes. Chem. Phys. Lipids 122 (1-2), 107-120.

(60) Dennison, S. R., Harris, F., and Phoenix, D. A. (2005) Are oblique orientated alpha-helices used by antimicrobial peptides for membrane invasion? Protein Pept. Lett. 12 (1), 27-29. 